\title{
Automated System for Detecting Neonatal Brain Injuries
}

\section{Rehan Ahmed}

\author{
Dept. of Electrical and Electronics Engineering, UCC
}

"The most dangerous journey a human ever makes in his life is from the womb of his mother to the outer world." (Lord Robert Winston)

The birth of a baby is a moment, enjoyed by many parents. However, in some rare cases these moments get shadowed due to the poor health of the new-born. Almost 1 in 5 pregnancies gets complicated and around $10 \%$ of all babies require admission to the special care unit due to problems of delivery.

Brain injury at the time of birth, due to lack of oxygen or blood in the brain, may cause neural dysfunctions or death in severe cases. In fact, it accounts for a third of all neonatal deaths globally and is the most common cause of long term severe neurological disability in neonates. Therefore, it is very important to diagnose and then treat the neonates with these brain injuries as early as possible.

a)

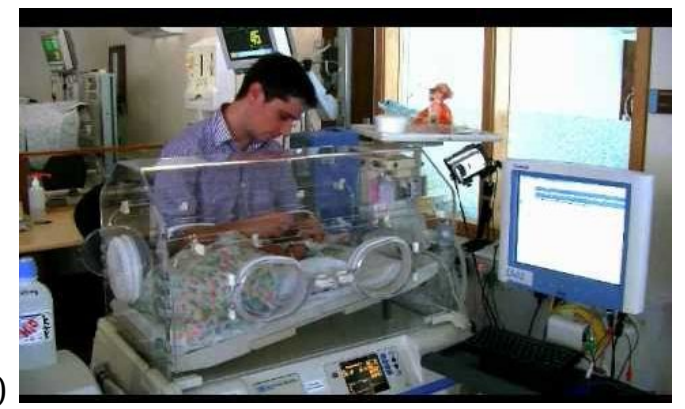

b)

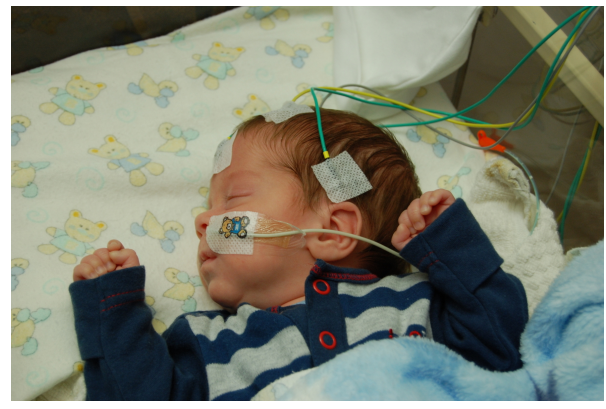

Figure 1: (a) A neonate being monitored in the NICU (Source: A shot from publicly available video of neonatal brain research group (https://youtu.be/aEtpIY8Wiy4?t=119)). (b) Electrodes placed on a neonate's head to record the EEG. Source: Neonatal brain research group, UCC, Cork

The problem with detecting brain injuries in neonates is that, in most cases the babies do not show any clear physical signs of the undergoing brain damage. In order to find such hidden brain injuries, the electrical activity of the brain is monitored using a method called Electroencephalography. Tiny electrodes (Figure 1b), are attached on the neonate's head to record the brain signals known as Electroencephalogram (EEG). EEG is considered the gold standard for detecting the timing and nature of neonatal brain injuries. Moreover, it also helps in monitoring any progression or improvement in the brain injury after the treatment is initiated. 
The normal EEG is a very random signal without any obvious pattern (Figure 2). EEG becomes abnormal when it loses its natural chaotic behaviour and a certain pattern starts to appear.

Clinically, EEG is visually analysed to find any abnormal patterns. Typically, an EEG recording can last for more than 24 hours. The neurologist analyses a 30 seconds window at a time and then proceeds to the next window. Given this scale, a 3 hour EEG recording equates to the length of a football stadium. Furthermore, the expertise to interpret the neonatal EEG is very scarce in busy Neonatal Intensive Care Unit (NICU). Thus, an automated system to detect brain injuries in neonates could significantly help clinical staff in diagnosis and to suggest an early treatment.

\section{Brain injuries in question}

This work investigates the automated system for the two most common neonatal brain injuries; Hypoxic Ischemic Encephalopathy (HIE) and seizures. HIE is the primary injury whereas seizure is the secondary injury caused either by HIE or some other brain problem. The reason for working on them at first is that they require the most urgent treatment in the NICU.

HIE is caused due to lack of oxygen (hypoxia) or impaired blood (ischemia) flow to the brain. The long-term outcome depends on the severity of the initial HIE insult. HIE is generally graded into four grades (Figure 2b.1). It can be seen, that the HIE effected EEG exhibits various patterns, some of which may be similar across HIE grades; however it is the inter-pattern variability or the way such patterns occur over the whole EEG recording which characterize its grade.

The treatment involves, cooling the infant to a body temperature of between $32-34^{\circ} \mathrm{C}$ for 72hours without interruption. However to be effective, it must be commenced within 6 hours of birth. In this narrow window of time the population of neonates who would benefit from treatment must be accurately identified.

Seizures are brief events due to abnormally excessive or synchronous neuronal activity in the brain. On the EEG recording seizures are defined as sudden, repetitive, evolving stereotyped waveforms that have a certain start and ending. Figure $2 \mathrm{~b} .2$ shows an example of a seizure pattern. Anti-epileptic drugs are used for the treatment. If seizures are not detected as early as possible then the resulting lack of treatment could cause a severe brain damage or death.

\section{Overview of the automated system for detecting brain injuries}

Figure 3 show an overview of the automated system to detect the brain injury in the neonatal EEG. First, the raw EEG is chunked into short segments (epochs) from which 


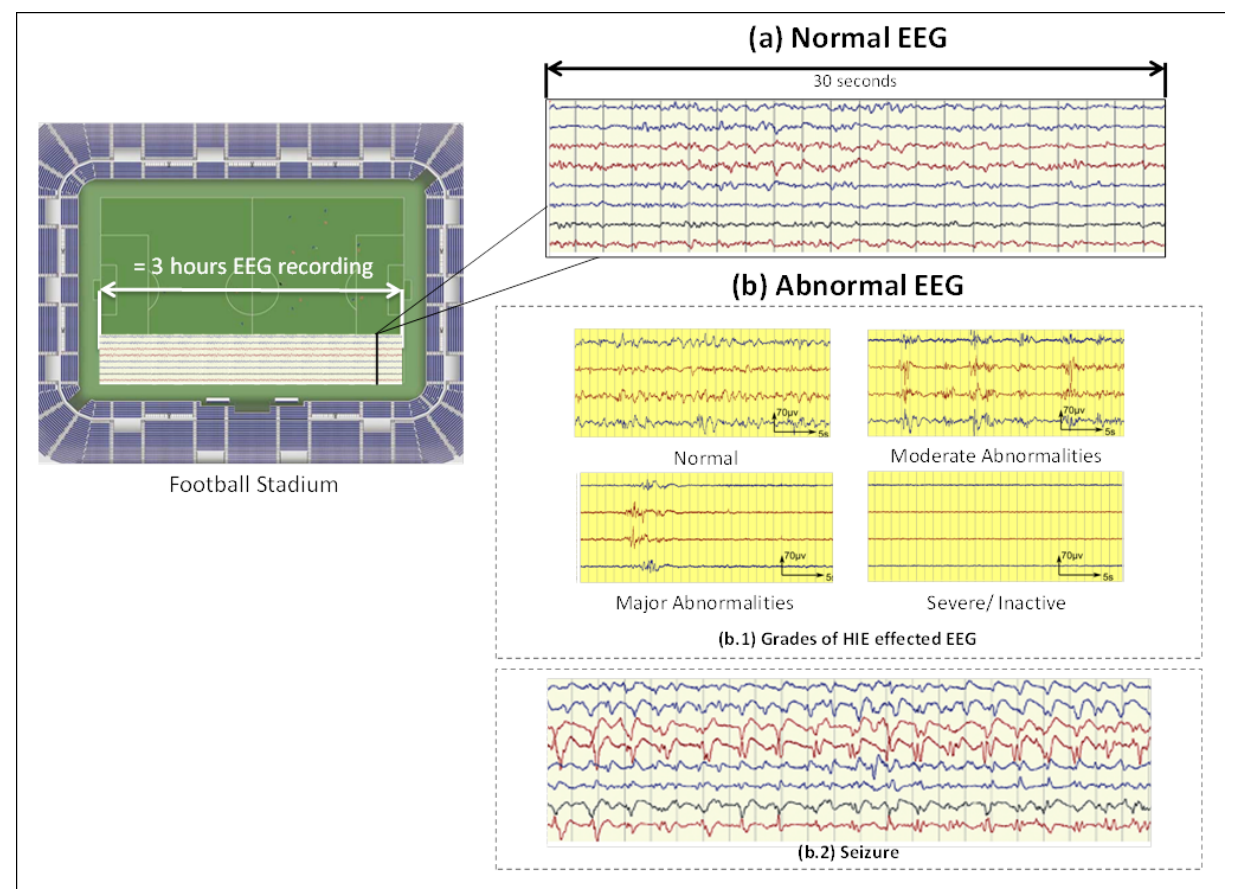

Figure 2: Examples of Normal and Abnormal EEG. Source: Author

different EEG features are extracted. A feature is a measurable attribute of a signal e.g. number of peaks in the analysis window or the mean amplitude of the signal. The features of this segment are then fed to a classifier which decides the class (normal/abnormal) of this epoch. A classifier makes a decision based on the data it is trained on. Figure 3 also highlights an illustration of a simple machine learning classifier. Here working of a support vector machine classifier is presented. During the training process it tries to find a hyper-plane that best separates the data of two classes. In the test/classification phase it calculates the distance from the separating hyper-plane to decide the class of a given test datapoint. The classifier's decision is later converted into a probability of the severity of the brain injury to make it easier for the clinicians to understand the decisions of this system.

\section{Exploring the contextual information to detect seizures}

It is well known that there is a lot of information in brain signals if looked over a longer time scale. For example, in the case of seizures, although there is a definite stereotypical pattern of this injury; these patterns evolve in frequency and shape through time. An example of such a seizure is shown in Figure 4. Similarly, for classifying the severity of HIE injury, it is necessary to not only detect the short events like a burst of high frequency activity but also to capture the slow variation of these events in time. Moreover, a clinical expert also analyses the EEG recordings by looking at the bigger picture. S/He goes back and forth through the recording to see what happened before and after a particular event, in order to define its class. 


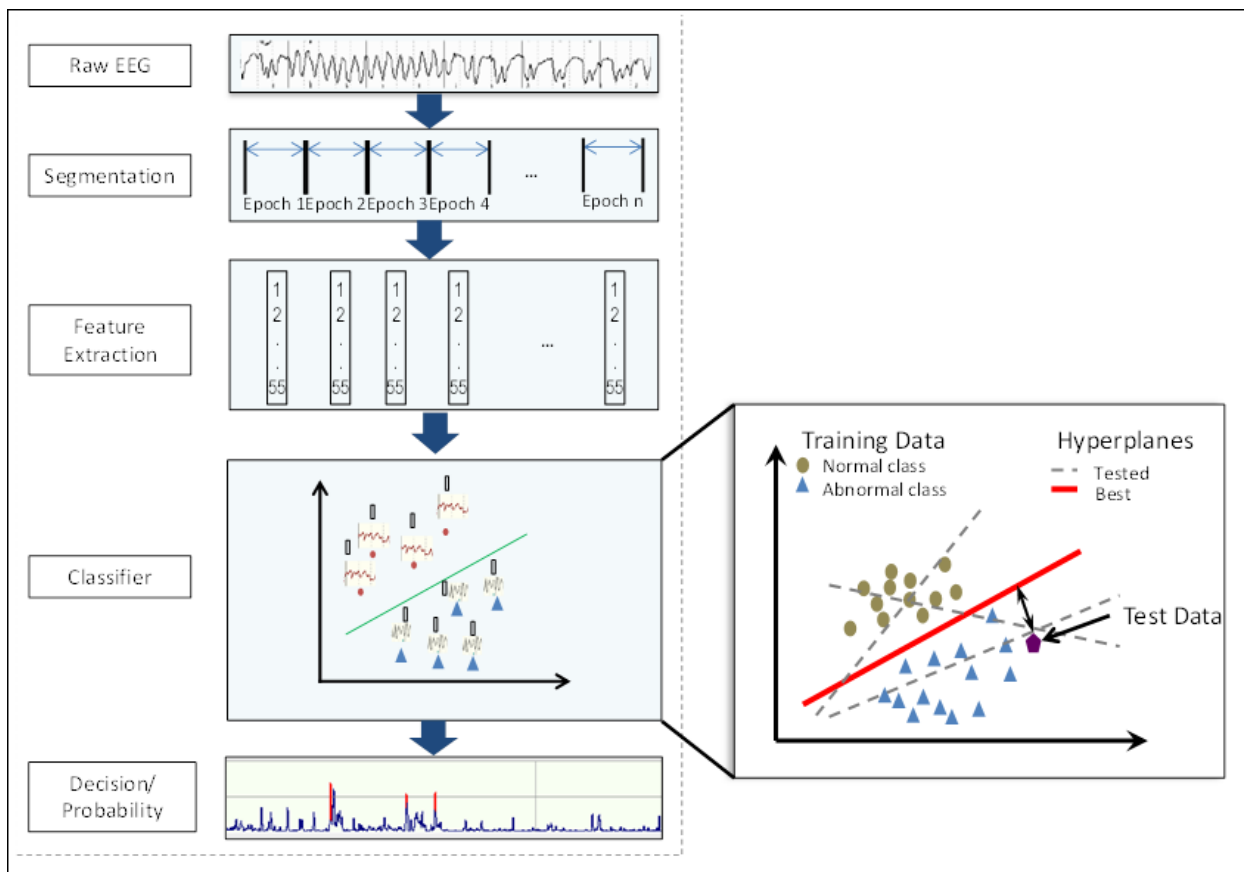

Figure 3: An overview of the automated system for brain injury detection. Source: Author.

A lot of work has been done in the past to develop automated systems for the detection/classification of brain injuries in neonates. However, the classifiers used inside most of these systems, were only able to classify discrete short segments of the EEG. Hence, these systems were either not able to capture the slower time varying changes in brain injuries or they used methods, before or after the classifier, that were not sophisticated enough to use this information to full extent. An example of such system is state of the art neonatal seizure detection system developed by our research group. Features were extracted from 8 seconds epochs of EEG and then classified by a SVM classifier. The contextual information was explored at the decision level by just taking an average of 30 seconds of past and future decisions of the classifier to decide the seizure probability of a given EEG epoch. Nonetheless, this system produced the best performance on the largest available neonatal dataset for seizure detection but its performance was poor in detecting short seizure events because they were being suppressed by this averaging operation.

Similarly, in the case of systems for automated grading of EEG recordings for classifying the HIE injury; the contextual information was explored by extracting features from a longer EEG epoch of 1 minute. It also misclassified many recordings because the features were not able to comprehend all the slower time varying events.

In our current work, a novel automated system is proposed that can look at this subtle but significantly important contextual information at the classifier level. Figure 5 shows an overview of the proposed sequence based classifier. It can be seen that all the modules of the system remain the same, except that another stage is added where features are combined together in sequences and then fed to the classifier. The classifier uses both the information extracted from short EEG epochs and also takes into account the temporal 


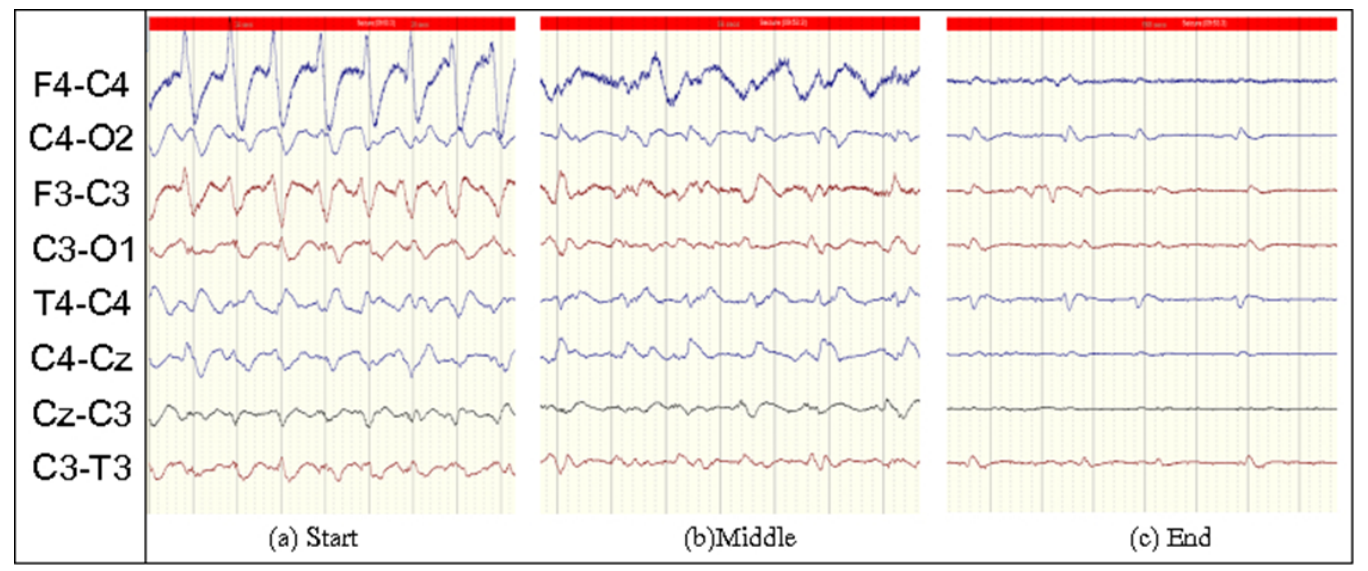

Figure 4: Changes in a seizure pattern in time. Source: Author

evolution of these features in a given sequence. In the example shown in Figure 5, a sequence of 3 epochs is used; however the system is able to cope with any sequence length. Hence we can call it a dynamic classifier based system.

The results show promising performance improvements in the detection of seizures. The system has not only helped in increasing the detection rate of both long and short seizures but has also reduced the number of false alarms. A total of $8 \%$ increase in detection rate was observed given 1 false alarm per hour.

We employed a similar system for grading the severity of HIE injury in one hour long EEG recordings. The system was able to classify the files into four grades with $87 \%$ accuracy. This was a $10 \%$ increase from the previous state of the art reported system.

\section{Conclusion and future work}

The methods investigated in this work are an important step forward towards the creation of highly intelligent automated systems to detect brain injuries which may help revolutionize neurocritical care in the NICU. Such systems will be able to explore not only the local contextual information of a single neonatal brain injury event but also the bigger global picture where information from other physiological signals e.g. heart rate, blood pressure will also be exploited to produce a score of neonate's overall brain health.

I would like to thank my supervisors, Dr. Gordon Lightbody, Professor Liam Marnane and Dr. Andriy Temko for the support and guidance in writing this article and throughout my PhD journey. Last but not the least, I would like to thank my wife Amna Shafqat who proof read and guided me to write this article for a non-specialist audience. 


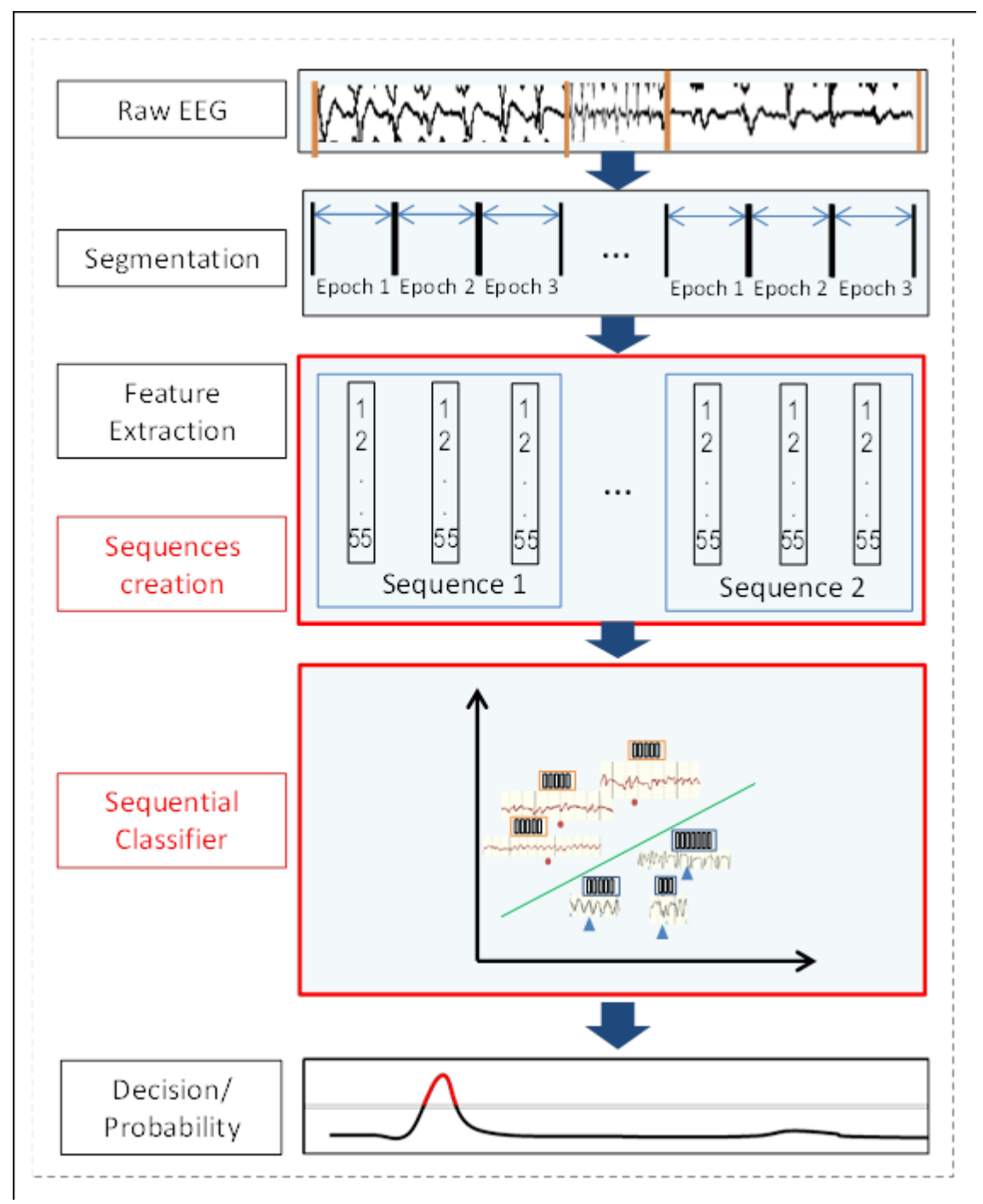

Figure 5: The proposed sequence classification based system. Source: Author 